\title{
High Precision Stokes Polarimetry for Scattering Light using Wide Dynamic Range Intensity Detector
}

\author{
Shuhei Shibata ${ }^{1, a}$, Yoshio Hayasaki ${ }^{1}$, Yukitoshi Otani ${ }^{1,2}$ and Toyohiko Yatagai ${ }^{1}$ \\ ${ }^{1}$ Center for Optical Research and Education, Utsunomiya University, 7-1-2 Yoto, Utsunomiya, Tochigi, Japan \\ ${ }^{2}$ Depertment of Optical Engineering, Utsunomiya University, 7-1-2 Yoto, Utsunomiya, Tochigi, Japan
}

\begin{abstract}
This paper proposes a Stokes polarimetry for scattering light from a sample surface. To achieve a high accuracy measurement two approaches of an intensity detector and analysis algorism of a Stokes parameter were proposed. The dynamic range of this detector can achieve up to $10^{10}$ by combination of change of neutral-density (ND) filters having different density and photon counting units. Stokes parameters can be measured by dual rotating of a retarder and an analyzer. The algorism of dual rotating polarimeter can be calibrated small linear diattenuation and linear retardance error of the retarder. This system can measured Stokes parameters from $-20^{\circ}$ to $70^{\circ}$ of its scattering angle. It is possible to measure Stokes parameters of scattering of dust and scratch of optical device with high precision. This paper shows accuracy of this system, checking the polarization change of scattering angle and influence of beam size.
\end{abstract}

\section{Introduction}

Recently there is a problem of dust and scratch of micrometer with nanometer order on an optical device such as a lens and a grating in manufacturing line. Currently specialists check samples with those problems. However it is difficult to discern dust and scratch even the specialist. One of discernment approach is a polarization measurement because polarization can have a sensitivity to measure surface condition with nanometers order. It is conceivable that discernment of dust and scratch become by measurement of Stokes parameters defined all polarization state. However it is necessary to develop an equipment of Stokes parameters measurement with very high precision for this discernment. One of approach for high precision measurement is development of intensity detector with high sensibility and high dynamic range. Our group proposed the detector having wide dynamic range detected intensity [1]. The dynamic range of most common detector is from $10^{3}$ to $10^{5}$. This detector can become $10^{10}$ of wide dynamic range using change of ND filters having different transmittance and photon counting units. By using only this intensity detector, reducing error of grating can be found by weak intensity between diffracted light distribution, and by comparing measurement and simulation of intensity distribution, shrink size of grating is possible to be estimated [2]. Second approach develops algorism of Stokes parameters measurement analysis of dual rotating method. Dual rotating method had been proposed calibration of retardance error of retardance such as quarter wave plate

\footnotetext{
${ }^{\mathrm{a}}$ Corresponding author: shibata_s@opt.utsunomiya-u.ac.jp
}

and half wave plate [3]. However some retarders have small diattenuation error. It is necessary to calibrate small diattenuation and retardance error for high accuracy measurement. The final pupas of research is to judge the dust and scratch by high accuracy polarimeter for scattering light using intensity detector with wide dynamic range and new algorism calibrated retardance error and small diattenuation error of retarder in dual rotating equipment. This paper shows a theory of calibration of those errors of retarder, to check the accuracy of Stokes parameters using this detector and to check the polarization change of scattering angle and influence of beam size.

\section{Stokes polarimetry by using wide dynamic range intensity detector}

\subsection{Intensity detector with wide dynamic range}

Figure 1 shows the schematic of this detector composed multiple ND filters and photon counting units.

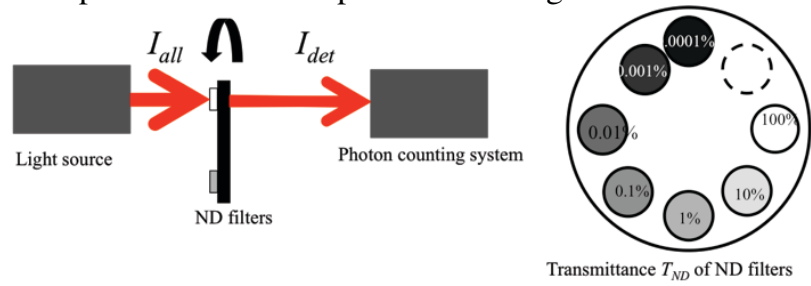

Figure 1 Intensity detector with wide dynamic range

If too mush photon numbers come to photon counting units, intensity reduces by ND filter knowing 
transmittance value until possible to detect by photon counting units. Before reduced intensity $I_{\text {all }}$ can be recognized using detected intensity $I_{\text {det }}$ by photon counting and transmittance $T_{N D}$ of ND filter in following equation,

$$
I_{\text {all }}=\frac{I_{d e t}}{T_{N D}}
$$

\subsection{Dual rotating Stokes polarimeter}

Figure 2 shows optical setup of dual rotating Stokes polarimeter. It is composed retarder having retradance $\delta$, axis transmittance ratio $\gamma$ related small diattenuation value and initial azimuthal angle $\theta_{c}$, analyzer of initial azimuthal angle $\theta_{p}$ and photon counting system as detector. The Stokes parameters can be measured by dual rotating retarder and analyzer with rotation ratios $\alpha$ : $\beta$. This polarimeter have to get number $\omega$ of intensity for measurement.

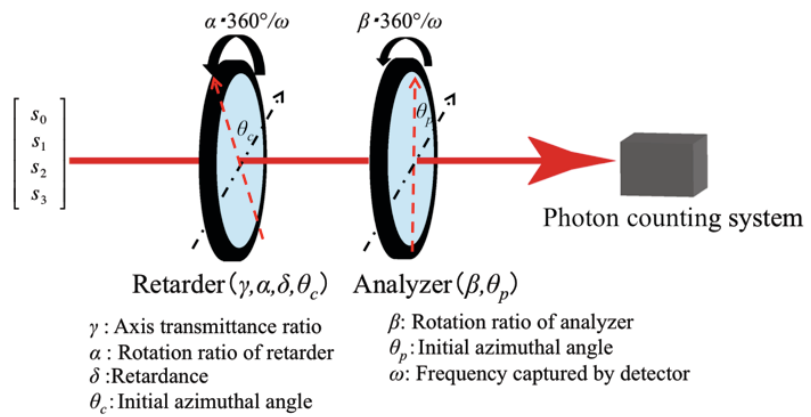

Figure 2 Dual rotating Stokes polarimter

Detected intensity $I$ by detector is given from incidental Stokes parameters $\left[s_{0}, s_{1}, s_{2}, s_{3}\right]$ and Muller matrixes among all polarization components,

$$
\begin{aligned}
I= & I_{0}\left[1+a_{2(\alpha-\beta)} \cos 2\left\{(\alpha-\beta)+\left(\theta_{c}-\theta_{p}\right)\right\}+\right. \\
& b_{2 \omega} \sin 2\left\{(\alpha-\beta)+\left(\theta_{c}-\theta_{p}\right)\right\}+a_{2(\alpha-\beta)} \cos 2\left\{(\alpha-\beta)+\theta_{p}\right\}, \\
& +b_{2(\alpha-\beta)} \sin 2\left\{(\alpha-\beta)+\theta_{p}\right)+a_{2 \alpha} \cos 2\left(\alpha+\theta_{c}\right) \\
& +b_{2 \alpha} \sin 2\left(\alpha+\theta_{c}\right)+a_{2(2 \alpha-\beta)} \cos 2\{(2 \alpha-\beta) \\
& \left.+\left(2 \theta_{c}-\theta_{p}\right)\right\}+b_{2(2 \alpha-\beta)} \sin 2\left\{(2 \alpha-\beta)+\left(2 \theta_{c}-\theta_{p}\right)\right\}
\end{aligned}
$$

where, each coefficient of frequency $\alpha$ and $\beta$ shows,

$$
\begin{aligned}
& a_{2(\alpha-\beta)}=-\frac{1-\gamma^{2}}{1+\gamma^{2}} \\
& a_{2 \beta}=\left[1+\frac{2 \gamma \cos \delta}{1+\gamma^{2}}\right] s_{1} \\
& a_{2 \alpha}=-\frac{1-\gamma^{2}}{1+\gamma^{2}} s_{1} \\
& a_{2(2 \alpha-\beta)}=\left[1-\frac{2 \gamma \cos \delta}{1+\gamma^{2}}\right] s_{1} \\
& b_{2(\alpha-\beta)}=-\frac{2 \gamma \sin \delta}{1+\gamma^{2}} s_{3} \\
& b_{2 \beta}=-\left[1+\frac{2 \gamma \cos \delta}{1+\gamma^{2}}\right] s_{2} \\
& b_{2 \alpha}=-\frac{1-\gamma^{2}}{1+\gamma^{2}} s_{2} \\
& b_{2(2 \alpha-\beta)}=-\left[1-\frac{2 \gamma \cos \delta}{1+\gamma^{2}}\right] s_{2}
\end{aligned}
$$

First it is necessary to measure initial azimuthal angle of retarder and analyzer for alignment. Those angles can be calculated as phase of each frequency of equation (2) using Fourier analysis in following equations,

$$
\begin{aligned}
& \tan 2 \theta_{p}=\frac{b_{2(\alpha-\beta)}}{a_{2(\alpha-\beta)}}, \\
& \tan 2 \theta_{p}=\frac{b_{2(\alpha-\beta)}}{a_{2(\alpha-\beta)}} .
\end{aligned}
$$

If Fourier coefficients equaled coefficients of Equation (3) of each frequency can be get by Fourier transformation, unknown Stokes parameters can be given from,

$$
\begin{gathered}
s_{1}=\frac{a_{2 \beta}+a_{2(2 \alpha-\beta)}}{2}, \\
s_{2}=-\frac{b_{2 \beta}+b_{2(2 \alpha-\beta)}}{2}, \\
s_{3}=-\frac{1+\gamma^{2}}{2 \gamma \sin \delta} b_{2(\alpha-\beta)} .
\end{gathered}
$$

The axis transmittance ratio $\gamma$ and retardance $\delta$ of retarder can concurrency be calculated from,

$$
\begin{gathered}
\gamma=\sqrt{\frac{1+a_{2(\alpha-\beta)}}{1-a_{2(\alpha-\beta)}}}, \\
\delta=\cos ^{-1}\left[\frac{1+\gamma^{2}}{2 \gamma} \cdot \frac{a_{2 \beta}-a_{2(2 \alpha-\beta)}+b_{2 \beta}-b_{2(2 \alpha-\beta)}}{a_{2 \beta}+a_{2(2 \alpha-\beta)}+b_{2 \beta}+b_{2(2 \alpha-\beta)}}\right] .
\end{gathered}
$$

This Stokes polarimeter can calibrate linear diattenuation and retardance of retarder by substituting from Equations (9) and (10) to equitation (8).

\section{Equipment of Stokes polarimetry for scattering light by using wide dynamic range intensity detector}

Figure 3 shows an equipment of Stokes polarimetry for scattering light by using wide dynamic range intensity detector. This system is composed by a laser diode LD of wavelength $635 \mathrm{~nm}$, the ND filter and photon counting system for expanding dynamic range of detected intensity, a half wave plate for controlling intensity, a polarizer as generated polarization, a sample, two pinholes for preventing back reflection and internal scattered light and quarter wave plate and polarizer for measurement of Stokes parameters after sample. Rotation ratio $\alpha$ : $\beta$ of this polarimeter is 3: 2. This equipment can rotate Stokes polarimeter system from $-70^{\circ}$ to $20^{\circ}$ and sample from $180^{\circ}$ to $180^{\circ}$ for scattered and reflected light measurement.

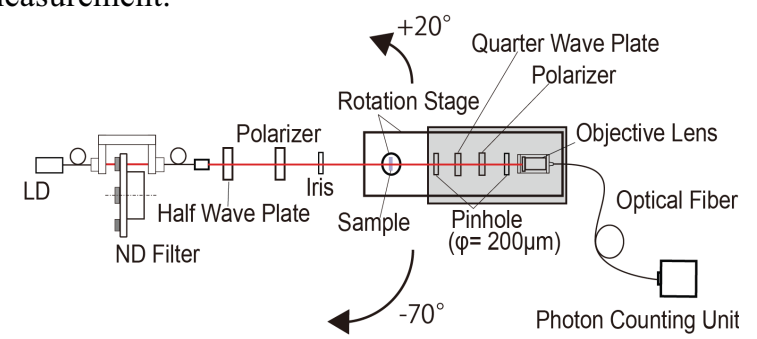

Figure 3 Equipment of Stokes polarimetry for scattering light by using wide dynamic range intensity detector 


\section{Results of Stokes polarimetry for scattering light by using wide dynamic range intensity detector}

\subsection{Checking accuracy of Stokes polarimeter}

Table 1 shows measurement results of the horizontal linear polarization, the $45^{\circ}$ linear polarization and right the circular polarization as typical polarization states in transmission type system. Those linear polarizations were made by a Glan-Thompson prism. The circular polarization is made by the Glan-Thompson prism and a quarter wave plate. However perfect circular polarization can't be created because the quarter wave plate usually has error of retardance. In this time Stokes parameters of theoretical circular polarization were calculated by using retardance measured by the AxoScan Mueller polarimeter produced by Axometrics, inc. Generally the accuracy of Stokes polarimeter is less than 0.01 . Measurement results are average of 20 measurement times. This experiment is captured $\omega=144$ times by photon counting units. The error of those Stokes parameters were gotten less than 0.003. Dispersion of this Stokes polarimeter with transmission type was gotten less than \pm 0.0002 from 20 times measurement.

Table 1 Results of the typical polarization states

\begin{tabular}{c|cccc}
\hline $\begin{array}{c}\text { Polarization } \\
\text { State }\end{array}$ & $\begin{array}{c}\text { Ideal } \\
\text { value }\end{array}$ & Result & Error & Dispersion \\
\hline $\begin{array}{c}\text { Horizontal } \\
\text { linear } \\
\text { polarization }\end{array}$ & {$\left[\begin{array}{l}1 \\
1 \\
0 \\
0\end{array}\right]$} & {$\left[\begin{array}{c}1 \\
1.002 \\
0.001 \\
0.000\end{array}\right]$} & 0.002 & \pm 0.00016 \\
$\begin{array}{c}45^{\circ} \text { linear } \\
\text { polarization }\end{array}$ & {$\left[\begin{array}{l}1 \\
0 \\
0 \\
1\end{array}\right]$} & {$\left[\begin{array}{c}1 \\
0.001 \\
1.002 \\
-0.002\end{array}\right]$} & 0.002 & \pm 0.00015 \\
$\begin{array}{c}\text { Right } \\
\text { circular }\end{array}$ & {$\left[\begin{array}{c}1 \\
1 \\
-0.10 \\
0 \\
0.99\end{array}\right]\left[\begin{array}{c}1 \\
-0.012 \\
0.001 \\
0.987\end{array}\right]$} & 0.003 & \pm 0.00017 \\
\hline
\end{tabular}

\subsection{Results of Stokes parameters of scattering light}

Figure 4 shows Stokes parameters of scattering light from a glass slide. In this measurement, scattering angle is rotated each $0.2^{\circ}$ from $-20^{\circ}$ to $20^{\circ}$. Horizontal linear polarization was passed through the glass slide. The glass slide as sample is set perpendicularity along optical axis. Stokes parameters after passing glass slide was measured by captured $\omega=36$ times. The intensity of transmitted light could be gotten 10 digits by expand dynamic range of detector. Scattering intensity was detected from 2 digits to 4 digits before $-3^{\circ}$ after $3^{\circ}$. However the polarization didn't change in scattering angle between $\pm 20^{\circ}$. Next, figure 5 shows the polarization property such as azimuthal angle of polarization, Degree of polarization and Ellipticity calculated from Stokes parameters of the glass slide passed $45^{\circ}$ linear polarization in larger scattering angle. Polarization didn't change from $-30^{\circ}$ to $20^{\circ}$ of scattering angle. $45^{\circ}$ linear polarization rotated to horizontal linear polarization before $-30^{\circ}$. Linear polarization became a little elliptical polarization. However this polarization didn't get depolarization effect due to scattering.

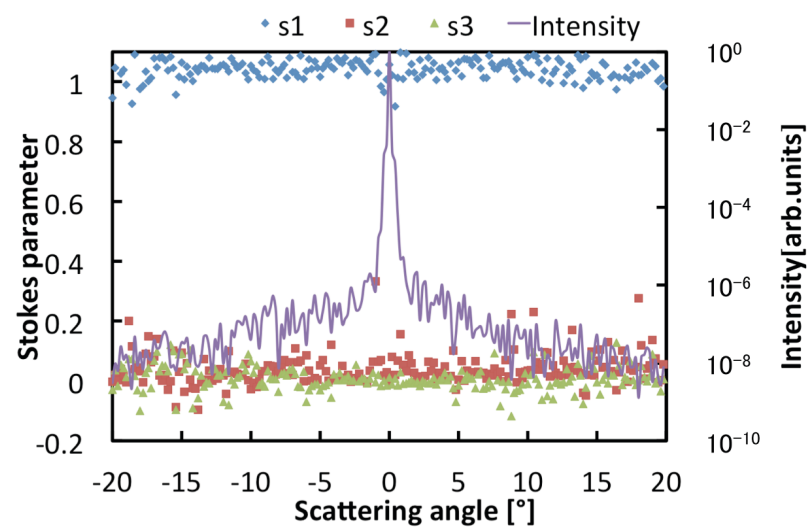

Figure 4 Result of Stokes parameters of scattering light

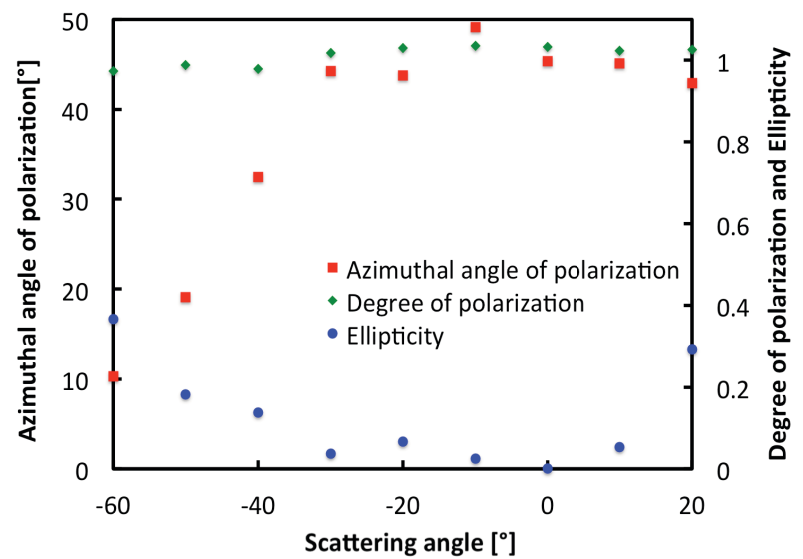

Figure 5 Polarization property of lager scattering angle

Figure 6 shows the degree of polarization depend on beam size changed 4 kind of pinhole size such as none, $500 \mu \mathrm{m}, 200 \mu \mathrm{m}$ and $100 \mu \mathrm{m}$. In this experiment vertical linear polarization was passed through the glass slide. Degree of polarization was gotten to degrease away from transmitted light without pinhole and in $500 \mu \mathrm{m}$ of pinhole size. However In $200 \mu \mathrm{m}$ and $100 \mu \mathrm{m}$ it didn't change. It is conceivable that this reason is to be intermixed many luminous fluxes has a little different polarization state. However in less than $200 \mu \mathrm{m}$ luminous flux was limited to some extent.

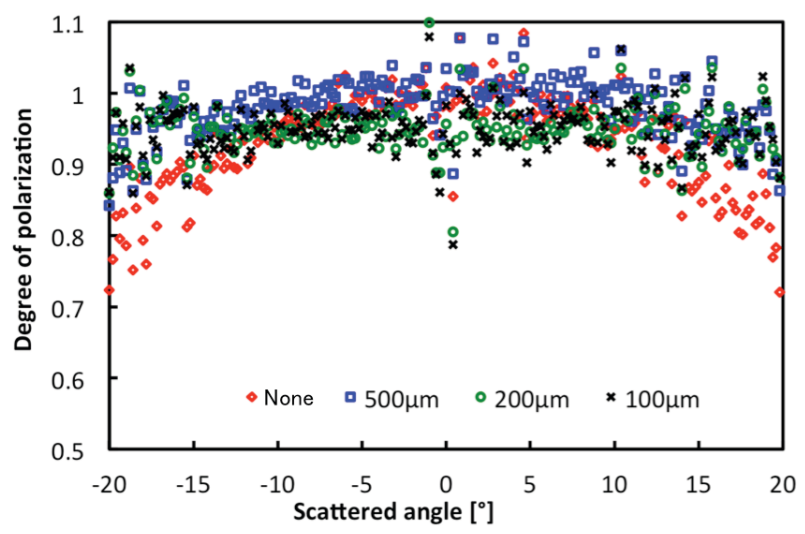

Figure 6 Degree of polarization depend on beam size 


\section{Conclusion}

Stokes polarimetry of light scattering light in high accuracy using wide dynamic range intensity detector using change of ND filters having different transmittance and photon counting units was proposed for discernment of dust and scratch. Stokes parameters can be measured in high precision by dual rotating method because it is possible to calibrate small diattenuation error and retardance of retarder. This paper showed the fundamental experiment. In transmission type the accuracy was gotten less than 0.003 by subtraction from theoretical value and \pm 0.0002 in variation of measurement. Stokes parameters of scattering light of glass slide was measured. From this result, it is understand that polarization state didn't change between $\pm 20^{\circ}$ of scattering angle, rotated to horizontal direction before $-30^{\circ}$ of scattering light. Degree of polarization kept less than $200 \mu \mathrm{m}$ of pinholes. However over than $500 \mu \mathrm{m}$ of pinholes it was decreased. In future, different surface roughness will be measured in this equipment for checking which level of roughness can keep a degree of polarization. Stokes parameters of dust and scratch of different size will be quantified for discernment of them.

\section{References}

1. T. Kiire,T. Meguriya, D. Barada, Y. Hayasaki, T. Yatagai, JJAP, 53, 2 (2014).

2. M. Hakko, T. Kiire, D. Barada, T. Yatagai, Y. Hayasaki, Optics Lett., 38, 19 (2013).

3. M. Tanaka, Y. Nakajima, H. Amamiya, M. Chujo, Y. Otani, Proc.SPIE, 7461(2009). 